Behavioural Sciences | James Hawdon

\title{
A new frontier for hate
}

\section{James Hawdon, Professor of} Sociology and Director of the Center for Peace Studies and Volence Prevention at Virginia Tech, USA, has spent many years studying online hate, other forms of cyberviolence. His work has examined how hate speech gets produced, how the hate groups and individuals that produce it become radicalised, how it spreads across the internet, what effects it has upon its producers and the targets societies can to do to prevent it.

Xtremism is as old as civilisation, but those advocating hate can spread their ideas more widely using the litemet. Bus mons of users now take part in a global llow of unformation and many ato ho, such as China, now see it as a political or cultural rival to orthodox power structures.

Over the last seven years, James Hawdon and his colleagues have examined online hate speech across seven countries that have high levels of Internet access and varying, but mostly low, levels of government restrictions, ranging from the almost absolute freedom of speech entrenched in US law, to countries with more robust anti-hate speech laws such as Germany and the UK.

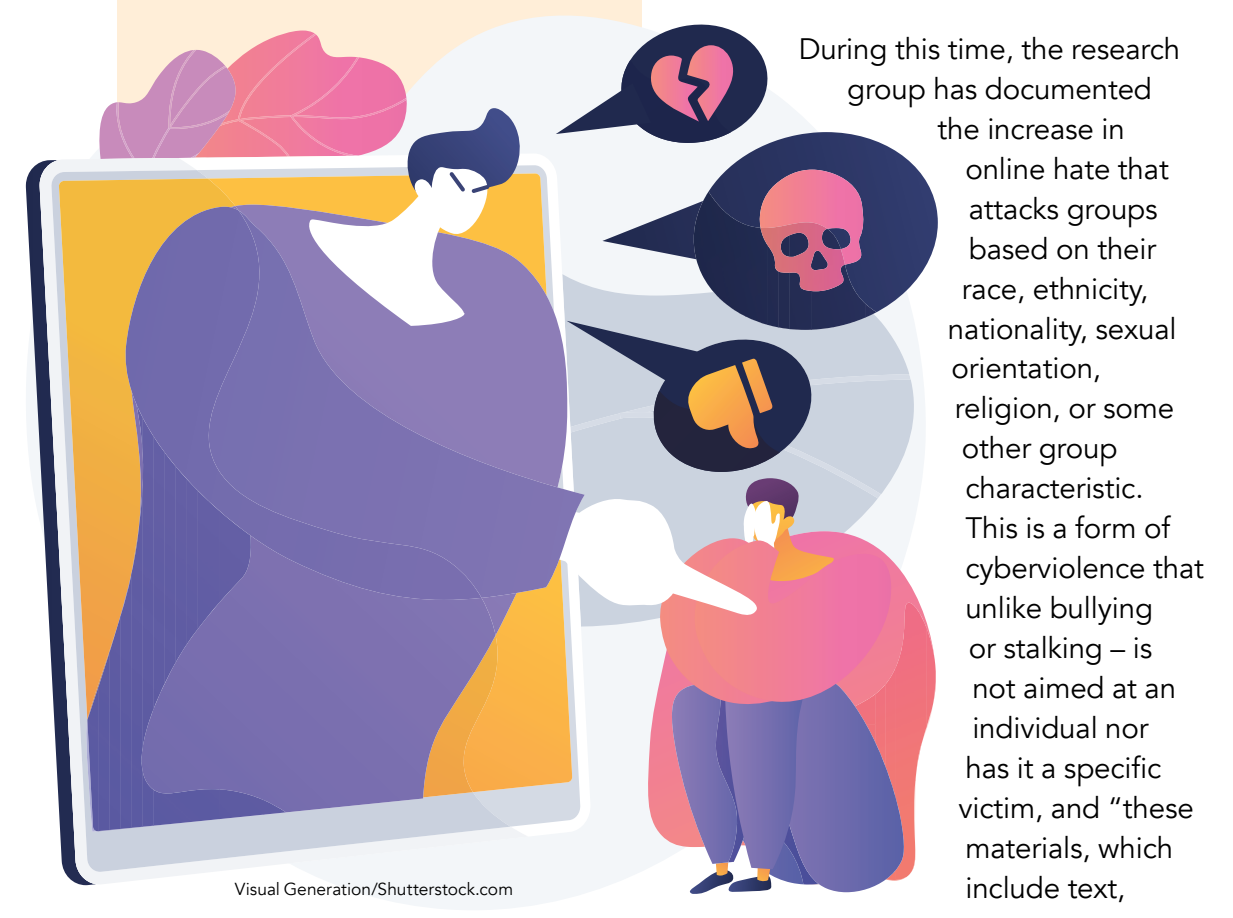

videos, photos, games, and other modes of communication, express

There have been many studies that confirm the negative impact of hate materials, and as one of Hawdon's collaborative works states, "This detrimental effects ... exposure to hate materials correlates with a range of negative behavioural and attitudin outcomes, such as decreased social trust, intergenerational transmissio of extremist ideology, reinforced discrimination against targeted groups, and fear and anger among members of a targeted group. Exposure to extremis acts of violence."

The most extreme example of this would be the rise of ISIS, and their to radicalise and recruit members and plan violent actions internationally. Up until recently, the majority of research into this topic has been conducted in English-speaking countries; however more recent studies are widening their scope and have included Finland, France, Germany, Poland, and Spain. Clearly the scope of research needs to be wider still given the increasing global nature of hate groups, and the possibility of other pan-national hate movements similar to ISIS rising up.

\section{WHO IS MAKING IT?}

There have been many studies by $D r$ Hawdon and others into who creates it, and what can be done to fight it.
Organised hate groups have had an ever-increasing presence on the Internet is ce th inception, but most online hate websites or commenting on socit media, and the numbers of people creating it has increased. For example, in one study in $2013,7 \%$ of Americans admitted to producing online hate; in $2018,21 \%$ admitted to doing so.

While there are many types of extremism, right-wing extremism currently dominates the Internet, or certainly the English-speaking part of it, and online hate producers tend to adopt an anti-cosmopolitan worldvie that sees their nation and culture as being under altack by immigration and multiculturalism. It also has misogynistic and anti-feminist tendencies, and it's no kind of hate materials than females.

There exist feelings of collective victimisation among members of these groups too, and those who feel a strong attachment to these online communities of like-minded individuals are also more likely to produce hate materials. Another factor is that there seems to be a correlation between these producers and self-control, as low self-control is an indicator of an inability to foresee the negative impacts on, and an insensitivity to, the consequences their behaviour could have themselves or the target of their hate.

\section{HOW HAS IT SPREAD?}

\section{dies demonstrate the}

processes of 'flocking' and 'feathering'. worldview 'flock' together, and once they do 'feathering' takes place where they learn and adopt the attitudes of other group members, says Dr Hawdon. This process can be amplified online as people join 'filter bubbles' that increase their contacts with likeminded people while limiting their exposure to alternative perspectives. It can reinforce negative worldviews that people then become comfortable spreading, the research group found out. As the number of sites spreading hate has grown, so has the number of people seeing $\mathrm{il}$, and the hate groups German ightwing ecremisto one

Exposure to Hate Materials

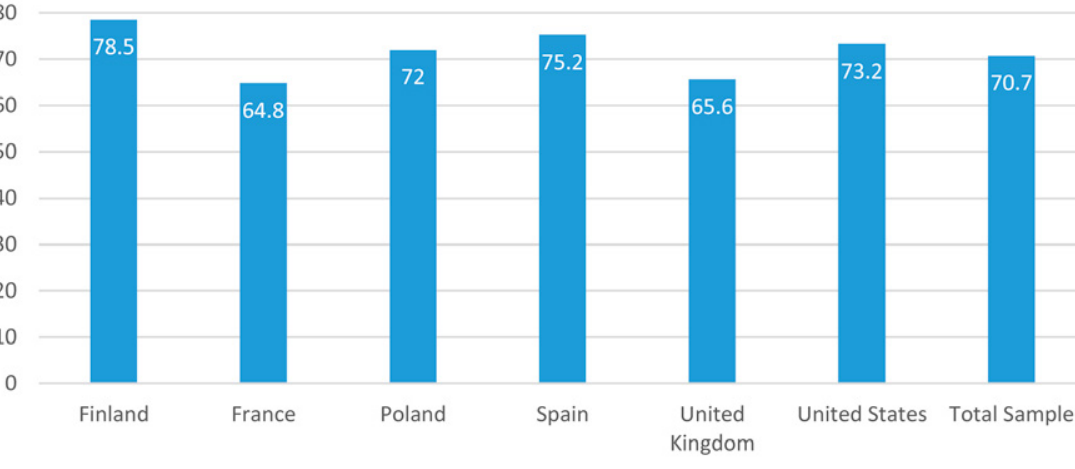

Percentage of respondents fro
writings or speech online

Online [right-wing] hate producers tend to adopt an anti-cosmopolitan worldview that sees their nation and culture as being under attack by

immigration and multiculturalism.

the Internet is 'the easiest way to make contacts and to take over and reputation and to advance'."

The situation is not helped by the algorithms that sites such as You Tube, Reddit and Facebook use, as these lecommend' things that users might like and can lead them to hate materia. consumed, they are also more likely

\section{Figure 2: Frequency of Exposure to Hate Materials in Six Nations}

Frequency of Exposure to Online Hate Materials

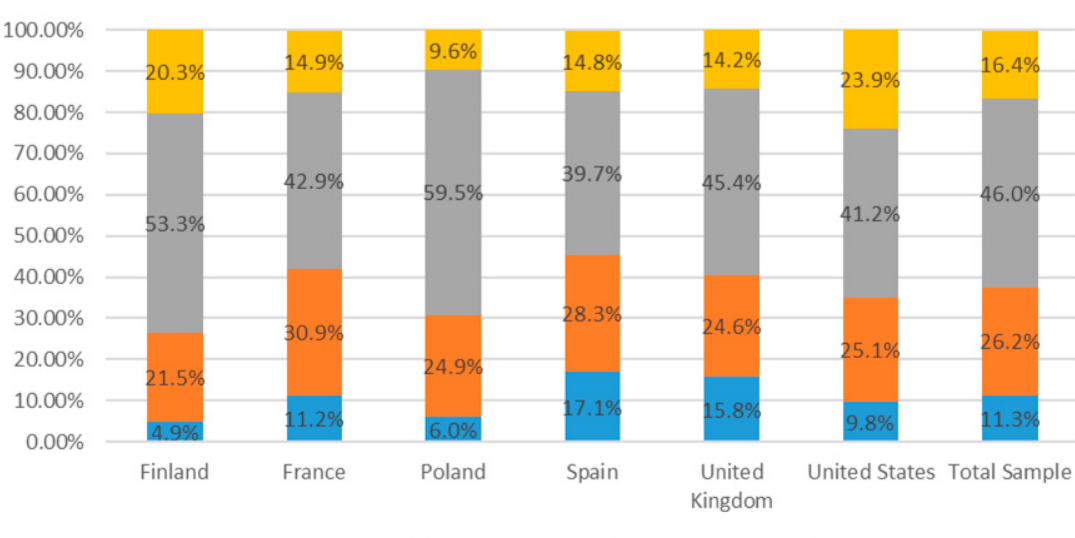

to be recommended again. They may to find a community and tighten the 'filter bubble'.

sers can also be exposed to materials (ang ' websies, such, or highly-libertarian websites, such as 4 Chan, which not only we bist such a 4 Chan, which not only

.


Platform where hate materials were seen or heard

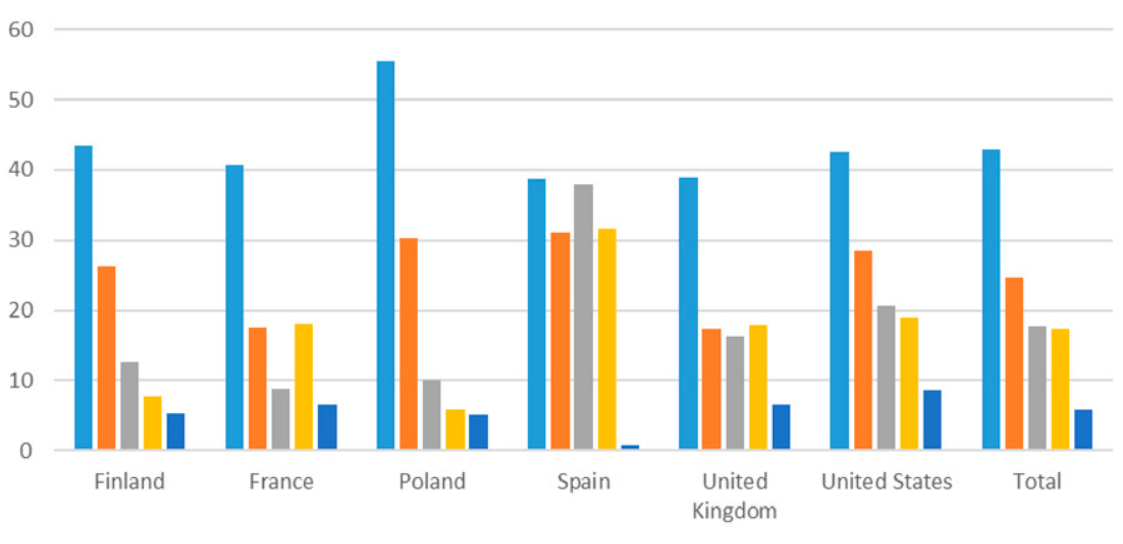

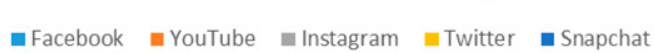

Internet plattorms where respondents viewed hate materials, including Facebook, YouTube,

posted) content. There is also evidence that younger people are more likely

an anti-cosmopolitan worldview all increase the chances of seeing ate materials if they are online hate.

forbidden by

their parents or

guardians, posst

Perhaps this online social control can convince extremists that, somewhat ironically, a tolerant society does not Exrism rioject, for example, uses to identify extremist threats and directly counter extremist ideology and recruitment. Similarly, Google's European division has announced plans for improving its ability to interrupt this process by using better machine learning to identify extremist videos, employing independent human flaggers to detect content that promotes hate activity, making and redirecting searches for radical content through anti-terrorism videos. However, freedom of speech protections can limit the effectiveness Chows as websites on a global network out of countries with the Social networking platforms may by removing messages, but the
sheer number of rebellion. tolerate extremist ideologies. platforms makes

One study
showed that the percentage of

We also see people use 'informal social Americans aged 18 to 25 who saw online hate increased from $53.9 \%$ in WHAT CAN BE DONE ABOUT IT? 2013 to $73.2 \%$ in 2018 and they found The most recent cross-national that people's online routines - spending studies suggest that enforcing antitime online, using multiple socia the hate speech laws may limit exposure one's social media networks, expressing also been steps taken by anti-hate controversial opinions online or having groups, social media and tech firms,

\section{Figure 4: How Respondents Arrived at Hate-Containing Site}

How Respondents Arrived at Hate-Containing Site

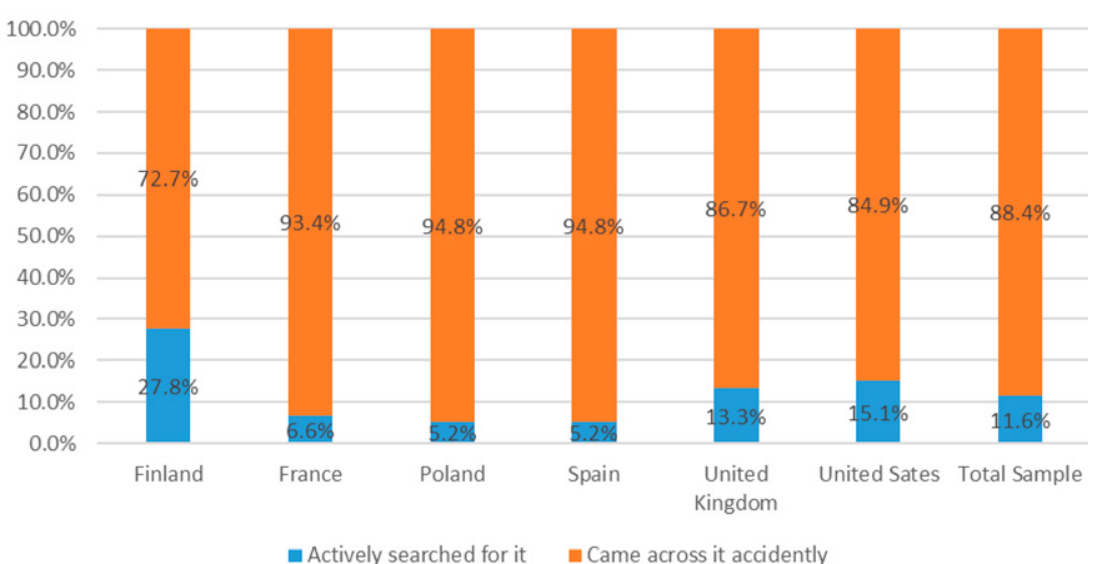

Percentage of respondents of
accidentally came across it. control' on people use 'firomal social pressure and will argue with posters or try to create a culture of anti-hate.

In Dr Hawdon's studies, over two-thirds of respondents reported that they tell people expressing hate online to stop suggests that perhaps this online social control can convine extremists that somewhat ironically, a tolerant society does not tolerate extremist ideologies. This may create a more tolerant virtual world, and, with luck, disrupt the radicalisation of the next perpetrator of hate-based violence.

However, given that most people are becoming more entrenched in their 'filter bubbles', and increasingly 'flock' to those with a shared worldview, this becomes more and more difficult over time. It's clear, therefore, that more jesearch needs to be done, and not just in the Western world and among and every age group.

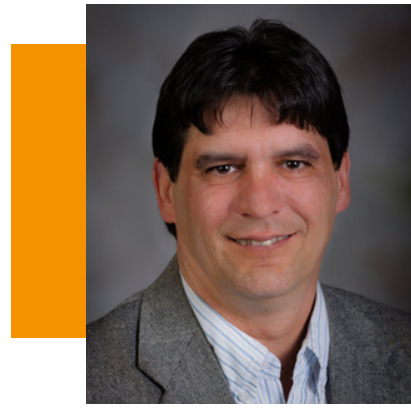

\section{Behind the Research}

\section{Dr James Hawdon}

E: hawdonj@vt.edu T: +1 $5402317476 \quad$ T: +15407501356

\section{Research Objectives}

Prof Hawdon studies how online hate spreads and what can be done to fight it.

\section{Detail}

James Hawdon

Center for
Prevention

Virginia Tech

495 Old Turner St.

205a Norris Hall

Blacksburg, Virginia 2406

USA

Bio

James Hawdon is Professor of

for Peace Studies and Violence

Prevention at Virginia Tech. His resea

focuses on how communities influence

the causes and consequences of violence. Recently, he has focused political polarisation, online hate, extremism, and cybercrime.

\section{Funding}

The research has been funded by

The Kone Foundation

The National Institute of Justice The Center froveace Studies and Virginia Tech's Institute for Society، Environment and Culture

\section{References}

- Reichelmann, A., Hawdon, J., Costello, M., Ryan, J.,
Blaya, C., Llorent, V., Oksanen, A., Räsänen, P., and Zych, l. (2020). Hate Knows No Boundaries: Online Hate in Six Nations. Deviant Behavior. Available at:

https://doliorg/10.1080/01639625.2020.1722337

- Hawdon, J., costello, M., and Bernatzky, C. (2019). CyberAdvocating Online Extremism. Social Forces, 98(1) 329-354. Available at: https://doi.org/10.1093/sf/soy115

- Costello, M., Barrett-Fox, R., Bernatzky, C., Hawdon, J., and Mendes, K. (2018). Predictors Youth Youth \& Socily Avaibung America's

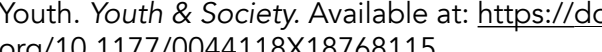

Exposure to online hate in four nations: A cross-nation consideration. Devant Behavior, 38(3), 254-266. Avallable - at: hattps://doi.org/10.1080/01639625.2016.1196985 extremism and online hate: Exposure among adolescents and young adults in four nations. Nordicom-Information, 37, 29-37.

- Hawdon, J., Oksanen, A., and Rásănen, P. (2014). Victim of online hate groups. American you h's exposure to online hate speech. In J. Hawdon, J. Ryan and M. Lucht . From bullies to terrorists (165-182). Lexington Books. - Hawdon, J. (2012). Applying differential association theory to online hate groups: A theoretical statement. Joun of Research on Finnish Society, 5, 39-47.

\section{Collaborators}

Matthew Costello (Clemson University); Pekka Räsänen Atte Oksanen (University of Tampere, Finland); Colin Bernatzky (University of California, Irvine); John Ryan (Virginia Tech); Ashley Reichelmann (Virginia Tech); Catherine Blaya (HEP du Canton Llorent (University of Cordoba

Spain): Izabela Zych (University of Cordoba): Thomas Ratliff (University of Pikeville)

\section{Personal Response}

What sparked your interest in cyberviolence? III Growing up in an industrial town watching crime became interested in how coal mines closed, 1 rimin behaviour. After studying how communities influence rates of violence and responses to violence in the the same theories that predict violence offline can af explain violence in the virtual communities in which we now spend so much time. This intrigued me because virtual communities provide an opportunity to test if our theories can be generalised and insights into how
they can be refined.

VIP VIRGINIA TECH 